\title{
KINETICS OF INTRAMOLECULAR AND \\ INTERMOLECULAR REACTIONS INVOLVING TWO FUNCTIONAL GROUPS ATTACHED TO POLYMERS
}

\author{
H. MORAWETZ
}

Department of Chemistry, Polytechnic Institute of Brooklyn, Brooklyn, N.Y. 11201, USA

\begin{abstract}
Three types of investigations are described. (a) When a polymer chain carries reactive substituents, the reaction rate may be increased by very large factors if these groups are sufficiently close to each other. More distant groups interact at rates governed by the chain flexibility. A technique is described for studying the effect of the excluded volume on the probability of cyclic chain conformations. (b) When reactive and catalytic substituents are attached to different polymer chains, their reaction should be hindered by the resistance of polymer coils to mutual interpenetration in good solvent media. Two theoretical approaches to this ' $k$ inetic excluded volume effect' are described. Experimental data do not conform to the expected behaviour and the reason for this discrepancy is unknown. (c) Crosslinked polyacrylamide beads carrying nucleophilic substituents were evaluated as catalysts for the solvolysis of nitrophenyl esters in a solution phase. Under certain conditions, groups attached to the gel were much more efficient catalysts than their analogues in homogeneous solution. This effect was ascribed to a cooperative action of two catalytic groups.
\end{abstract}

\section{INTRODUCTION}

It is a widely held generalization that functional groups attached to macromolecules have the same reactivity as their low molecular weight analogues. This is a useful concept as a starting point for the analysis of kinetic data involving reactions of polymeric reagents with low molecular weight species. If the polymer behaves very differently from its analogue, this difference may be the result of several factors. (a) The sensitive bond may be attached very close to the backbone of the polymer and the resulting steric hindrance may then not be easily simulated in a molecule of low molecular weight. This would account, for instance, for the well-known difficulty of the solvolysis of poly (methacrylic acid) esters. (b) The 'effective solvent medium' in the neighbourhood of a polymer chain may be significantly different from the solvent in bulk. This explanation has been invoked, for instance, to explain the fact that poly ( $N$-isopropylacrylamide) or poly( $N$-vinylacetamide) undergo isotopic hydrogen exchange much more slowly than their low molecular weight analogues ${ }^{1,2}$. (c) If a low molecular weight 
reagent is drawn into the domain of a macromolecule by long-range Coulombic interactions ${ }^{3,4}$ or by hydrophobic bonding ${ }^{5,6}$, the rate of its reaction with a group attached to the polymer may be many orders of magnitude above that characterizing an analogous process between two small molecules.

In this report I shall be concerned with a different class of effects resulting from the macromolecular nature of a chemical reagent, that is, with effects observed when two interacting groups are both appended to macromolecules. Three cases will be discussed, (a) reactions involving two interacting groups attached to the same chain backbone, (b) reactions involving two groups attached to different macromolecules in highly dilute solutions and (c) cooperative catalytic effects of groups attached to a crosslinked gel interacting with a small molecule.

\section{KINETICS OF REACTIONS INVOLVING INTERACTIONS OF TWO GROUPS ATTACHED TO THE SAME POLYMER CHAIN}

Intramolecular reactions of two groups carried by the same chain molecule are of two types. In the first class, interaction of groups carried by substituents of two neighbouring monomer residues of a vinyl polymer is heavily favoured since it leads to a 5-or 6-membered cyclic structure in the transition state. A good example of a process of this type is the hydrolysis of acrylic acid copolymers with small concentrations of an aryl acrylate or methacrylate. This reaction proceeds by the mechanism
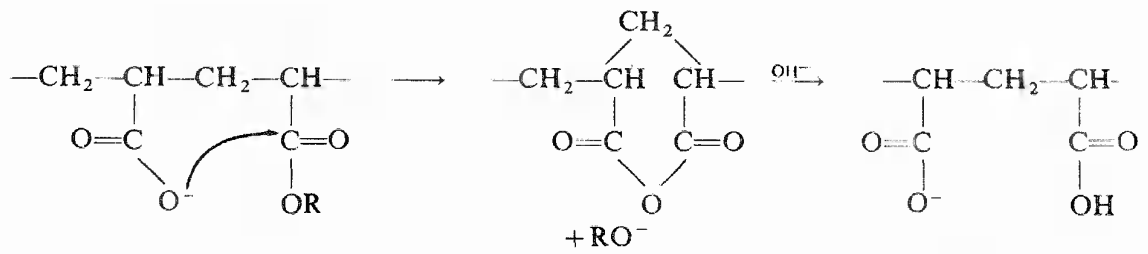

so that the solvolysis rate is exactly proportional to the fraction of the carboxyl groups which is ionized ${ }^{7}$. A comparison of this intramolecular process with the analogous bimolecular reaction, i.e. the attack of acetate on aryl esters leads to the following important observations:

(i) An intramolecular process corresponds to a very high 'effective local concentration', $C_{\text {eff }}$, of the carboxylate at the reaction site. For instance, the data of Gaetjens and Morawet $z^{7}$ show that the intramolecular process in phenyl succinate is 4500 times as fast as for phenyl acetate in 1 $\mathrm{M}$ acetate solution, i.e. $C_{\text {eff }}$ is 4500 .

(ii) The probability of a neighbouring group attack is highly sensitive to the size of the cyclic structure in the transition state and to a variety of steric restraints. For instance, glutaric anhydride is formed two orders of magnitude more slowly from glutaric monoesters than succinic anhydride from succinic monoesters ${ }^{7}$, and ester displacement from the monoester anion

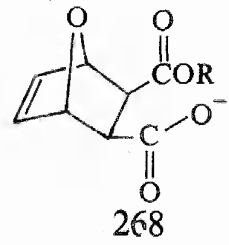


takes place 230 times as rapidly as for the corresponding succinic acid monoester ${ }^{8}$.

(iii) The ease with which the carboxylate can attack the neighbouring ester group may be highly sensitive to the relative steric configuration of the carbons to which the carboxylate and ester groups are attached. Thus, the solvolysis of methacrylic acid copolymers carrying a small number of aryl ester groups deviates strongly from first-order kinetics, since neighbouring group interactions in the sequences

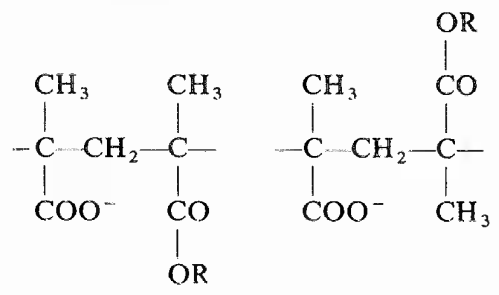

are characterized by rate constants differing by an order of magnitude ${ }^{9}$.

A very different situation arises if the potentially interacting groups are at a larger distance from one another so that their interaction would lead to larger cyclic structures. It is well known that $7-12$ membered ring formation involves large steric strain. For very large rings a theoretical estimate of the probability of a cyclic conformation may be obtained in the absence of an excluded volume effect. Using the model of a freely jointed chain with $\mathrm{Z}$ links of length $b$, this probability corresponds to an effective local concentration, $C_{\text {eff }}$, of the second chain end in the vicinity of the first chain end ${ }^{10}$

$$
\begin{aligned}
C_{\text {eff }}= & \left(\bar{N}_{\mathrm{A}} / 1000\right) \operatorname{Lim}_{h \rightarrow 0} W(h) \mathrm{d} h / 4 \pi h^{2} \mathrm{~d} h \\
= & \left(\bar{N}_{\mathrm{A}} / 1000\right)\left(3 / 2 \pi\left\langle h^{2}\right\rangle\right)^{\frac{3}{2}}
\end{aligned}
$$

Here $C_{\text {eff }}$ is expressed in units of mol $1^{-1}, \bar{N}_{\mathrm{A}}$ is Avogadro's number, $W(h)$ is the probability distribution function of the end-to-end displacement $h$ and $\left\langle h^{2}\right\rangle=Z b^{2}$ is the mean value of $h^{2}$. If two interacting groups are attached to the chain ends, the rate constant $k_{1}$ governing the intramolecular reaction will be

$$
k_{1}=\left(\bar{N}_{\mathrm{A}} k_{2} / 1000\right)\left(3 / 2 \pi\left\langle h^{2}\right\rangle\right)^{\frac{3}{2}} \equiv C^{\prime} Z^{-\frac{3}{2}}
$$

where $k_{2}$ is the second-order rate constant characterizing the analogous intermolecular reaction $\dagger$. For real polymer chains in $\theta$-solvents where $\left\langle h^{2}\right\rangle=C_{\infty} Z b^{2}$ (with the 'characteristic ratio' $C_{\infty}$ reflecting short-range interactions governing the chain flexibility ${ }^{11}$ ), equation 2 should be modified to

$$
k_{1}=C^{\prime \prime} Z^{-\frac{3}{2}}: \quad C^{\prime \prime}=C^{\prime} / C_{\infty}^{\frac{3}{2}}
$$

No corresponding analysis has been carried out for the rate of cyclization of chains if the excluded volume effect is operative. This will obviously

This treatment assumes that the cyclization has a substantial activation energy, so that the rate of the process is not controlled by the rate of conformational transitions of the polymer chain. 


\section{H. MORAWETZ}

decrease the probability of cyclic conformations and will lead to a steeper decline of $k_{1}$ with increasing $Z$. Computer generation of chains on a tetrahedral lattice has led to the estimate that $k_{1}$ should become proportional to $Z^{-2}$ when the excluded volume effect is large and the chains are long ${ }^{12}$ but no direct verification of the value of this 'chain closure exponent' exists.

Experimental studies designed to check the applicability of equation 1 for polymers in $\theta$-solvents or the dependence of the cyclization rate on chain length in better solvent media encounter formidable difficulties. For straightforward interpretation of experimental data we should require not only monodisperse polymers, with each chain carrying the two interacting groups at its two ends, but the kinetics of the interaction of these groups would have to be observed at dilutions such that the intermolecular process becomes negligible. Since it appears at present impossible to meet these conditions, we have approached the problem in an indirect manner ${ }^{13,14}$.

We prepared an acrylamide copolymer with a small proportion of a comonomer with a reactive side chain (a $p$-nitrophenyl ester) and a small proportion of a comonomer with a side chain containing a pyridine residue, which is known to catalyse the solvolysis of phenyl esters ${ }^{15}$. In analogy with equation 3 we would then expect the probability of solvolysis of a given ester group to be governed, in a $\theta$-solvent, by a rate constant of the form

$$
k_{1}=C^{\prime \prime} \sum_{i}^{\prime} x_{i}^{-\frac{3}{2}}
$$

where $x_{i}$ is the number of bonds separating a given ester group from the $i$ th catalytic pyridine residue attached to the same polymer chain. The summation excludes $x_{i}<x^{\prime}$, where $x^{\prime}$ is the number of bonds in the smallest ring which can form without significant strain. Since the catalytic groups are distributed at random along the chain, each ester will be characterized by a different value of $k_{1}$ and the kinetics of the solvolysis will be given by

$$
y(t)=\int W\left(k_{1}\right) \exp \left(-k_{1} t\right) \mathrm{d} k_{1}
$$

where $y(t)$ is the fraction of unreacted ester at time $t$ and $W\left(k_{1}\right)$ is the probability distribution function of $k_{1}$.

For any given density of catalytic groups along the polymer chain, deviation from first-order kinetics should increase with the breadth of the distribution function $W\left(k_{1}\right)$. This, in turn, should increase with an increase in the 'chain closure exponent' which, as we have seen, has been estimated to increase from a value of $\frac{3}{2}$ for chains with zero volume to a value of 2 when the excluded volume becomes large. It was the main purpose of our study to compare these estimates with experimental evidence.

In analysing kinetic data obtained with polymers carrying substituents on a fraction $\omega$ of the monomer units we used a computer simulation technique. We generated 100 chains containing 1000 units with a probability $\omega$ that any one unit was catalytic. The reactive group was placed on the central unit of the chain and with the assumption that the catalytic and reactive groups could interact only if separated by ten or more monomer residues, the time dependence of the reaction corresponding to a ring closure exponent $a$ was evaluated by 


$$
\begin{gathered}
k_{n}=C\left[\sum_{j=1}^{j=490} p_{n j}(500-j)^{-a}+\sum_{j=510}^{j=1000} p_{n j}(j-500)^{-a}\right] \\
y(t)=(1 / 1000) \sum_{n} \exp \left(-k_{n} t\right)
\end{gathered}
$$

Here $p_{n j}=1$ or $p_{n j}=0$ depending on whether the $j$ th residue of the $n$th chain is, or is not, catalytic and the constant $C$ is adjusted so as to make equation 7 yield the experimentally observed initial reaction rate. The procedure consists then in comparing the curvature of plots of $\log y$ against $t$ when using data computed with different ring closure exponents and the curvature characterizing a similar plot of experimental data. The kinetic pattern observed in a good solvent medium was found to be well fitted by data computed with $a=2$, that is, the ring closure exponent previously estimated for chains with a large excluded volume by generating chains on a tetrahedral lattice. On the other hand, when the solvent medium was adjusted to approach the $\theta$-condition, the chain closure exponent seemed to be about 1.6, distinctly higher than the $\frac{3}{2}$ value predicted from the model of a random-flight chain with zero volume.

Another interesting conclusion from this study is obtained from $k_{1}^{\text {init }} / k_{2} \omega$ where $k_{1}^{\text {init }}$ is the initial apparent first-order rate constant of the intramolecular process. This ratio may be identified as being equal to $C_{\text {eff }}^{\max }$ the effective local concentration of catalytic groups at the site of a reactive group in a chain containing a single reactive residue and catalytic groups carried by all the other monomer units. We found $k_{1}^{\text {init }} / k_{2} \omega$ to be in the range 2.7 $2.9 \mathrm{M}-\mathrm{a}$ very substantial value. It is about three times as high as $C_{\mathrm{eff}}^{\max }$, values estimated from association equilibria involving two groups attached to a randomly coiled polymer chain ${ }^{16}$.

\section{THE SEARCH FOR A 'KINETIC EXCLUDED VOLUME EFFECT'}

The large second virial coefficients characterizing the osmotic behaviour of flexible chain macromolecules in good solvent media have been interpreted as a consequence of a strong resistance of the molecular coils to mutual interpenetration. Some years ago, Morawetz and Song ${ }^{17}$ suggested that the large excluded volume implied by the thermodynamic behaviour of dilute polymer solutions should also lead to pronounced kinetic effects. Specifically it was argued that the reaction rate in a highly dilute solution of two similar macromolecules carrying a small number of reactive or catalytic substituents, respectively, should be much smaller, in a good solvent medium, than the reaction of low molecular weight analogues of the interacting species. Qualitatively, such a result seemed plausible since the molecular chains were unlikely to penetrate each other, so that only the reactive and catalytic groups near the 'surface' of the coiled macromolecules at any given time would be accessible to each other. This concept also led to the expectation that the observed rate constant would decrease rapidly with the length of the chain to which the interacting groups are attached. Surprisingly, experimental results with systems containing mixtures of acrylamide copolymers carrying a small number of $p$-nitrophenyl ester groups or catalytic imidazole residues failed to reveal the expected 'kinetic excluded volume effect'. 
We have now formulated a theory of this effect based on two models ${ }^{18}$ :

(i) In the first model the two macromolecules carrying reactive and catalytic substituents, respectively, are assumed to have the same molecular volume $v_{2}$ and their randomly coiled chains are represented by spherical clouds with a uniform chain segment density and a volume $v_{\mathbf{e}}$. Since the two polymers are very similar to each other, they are assigned the same polymer-solvent interaction parameter $\chi$, while the polymer--polymer interaction parameter is taken as zero.

If a solution with a volume $V$ contains one reactive and one catalytic polymer chain, the probability $W_{0}$ that a reactive group is, at any given time, within a volume $v_{j}$, jointly occupied by the two interpenetrating spheres is

$$
W_{0}=\frac{1}{v_{\mathrm{e}}} \frac{\int_{V} v_{j}(r) \exp [-\Delta G(r) / k T] 4 \pi r^{2} \mathrm{~d} r}{\exp [-\Delta G(r) / k T] 4 \pi r^{2} \mathrm{~d} r}
$$

where $r$ is the distance between the centres of the spheres representing the polymer coils and $\Delta G(r)$ is the increase in free energy resulting from the interpenetration of the two spheres. If the system contains $n_{\mathrm{c}}$ catalytic chains and only binary interactions need to be considered, this probability becomes $W=n_{\mathrm{c}} W_{0}$. Whenever a reactive group finds itself within a volume jointly occupied by the equivalent spheres of a reactive and a catalytic polymer, it is exposed to a local concentration $c_{l}$ of catalytic groups which is related to their stoichiometric concentration $c$ by $c_{l}=c\left(V / n_{\mathrm{c}} v_{\mathrm{e}}\right)$. The ratio of the secondorder rate constants $k_{2}$ and $k_{2}^{0}$ characterizing a reaction between groups attached to the two polymer chains and a reaction between their low molecular weight analogues should then be

$$
k_{2} / k_{2}^{0}=c_{l} W / c=\left(V / v_{\mathrm{e}}\right) W_{0}
$$

According to Flory ${ }^{19}, \Delta G(r)=2 k T v_{\mathrm{j}}\left(\frac{1}{2}-\chi\right)\left(v_{2} / v_{\mathrm{c}}\right)^{2} / v_{1}$ where $v_{1}$ is the molecular volume of the solvent. Since $\Delta G(r)=0$ for $r \geqslant 2 R_{\mathrm{e}}$, where $R_{\mathrm{e}}$ is the radius of the equivalent spheres, and the solution is assumed to be dilute, the denominator in equation 8 may be approximated by $V$. With $x=r / 2 R_{\mathrm{e}}$, $v_{\mathrm{j}}=v_{\mathrm{e}}\left(x^{3}-3 x+2\right) / 2$ for $x<1$ and $v_{\mathrm{j}}=0$ for $x \geqslant 1$,

$$
\begin{aligned}
k_{2} / k_{2}^{0} & =12 \int_{0}^{1}\left(x^{5}-3 x^{3}+2 x^{2}\right) \exp \left[-X\left(x^{3}-3 x+2\right) / 2\right] \mathrm{d} x \\
X & =\left(v_{2}^{2} / v_{1} v_{\mathrm{e}}\right)\left(\frac{1}{2}-\chi\right)
\end{aligned}
$$

This result leads to a rapid decay of the $k_{2} / k_{2}^{0}$ ratio with an increase in the parameter $X$. For instance, for $X=1,2,3$ and 4 the computed values for $k_{2} / k_{2}^{0}$ are $0.56,0.36,0.25$ and 0.19 .

(ii) A second approach to an analysis of the 'kinetic excluded volume effect' is based on the transition state theory. Let us consider two polymer chains with a reactive and a catalytic group attached to the central chain segment. It is assumed that the mixing of the two chains is thermoneutral. If we then compare the activation parameters for the formation of the transition state from the two polymeric reagents and their low molecular weight analogues, 
we find that the only difference is due to the decrease in configurational entropy when the two linear chains combine to a cruciform transition state. Specifically, this treatment leads to

$$
k_{2} / k_{2}^{0}=\Omega^{\ddagger} / \Omega^{2}
$$

where $\Omega^{\ddagger}$ and $\Omega$ are the conformational partition functions for the transition state, in which the two macromolecules have their central segments connected to each other, and the linear reagent molecules, respectively.

To get a feeling for the behaviour of $\Omega^{\ddagger} / \Omega^{2}$, we generated linear chains and chains connected by links of various lengths by a computer on a tetrahedral lattice. We assumed then $\Omega^{\ddagger} / \Omega^{2}=\left(f^{\ddagger}\right)^{2} / f^{2}$ where $f^{\ddagger}$ and $f$ are the fractions of non-selfintersecting chains representing the bridged transition state and the linear reagent molecules, respectively. The conclusions may be summarized as follows : (a) For long chains, the dependence of $k_{2} / k_{2}^{0}$ on the number $Z$ of links in the reagent chains is represented by $k_{2} / k_{2}^{0}=6.2 Z^{-0.56}$ and $k_{2} / k_{2}^{0}=7.0 Z^{-0.48}$ when the formation of the transition state involves 8 -link and 16-link bridges between the reagent chains. (b) If the interacting groups are attached to the chain end, rather than to the central chain segment, the variation of $k_{2} / k_{2}^{0}$ with $Z$ is much less pronounced, i.e. $k_{2} / k_{2}^{0}=1.2 Z^{-0.15}$.

The model involving specifically the chain character of the reagents and the bridged transition state seems much more realistic than the spherical cloud model. It is particularly useful in revealing the importance of the location along the chain molecule to which the interacting groups are attached. However, the simplification required for computer simulation, i.e. the neglect of the effect of side chains, of polymer-solvent interaction and of differences in the energy of different chain conformations, makes it impossible to compare the results quantitatively with experimental data.

At any rate, a recent experimental study ${ }^{19}$ did not bear out the theoretical predictions. Using a reactive acrylamide copolymer with $\bar{M}=1.24 \times 10^{5}$ and catalytic copolymers with $\bar{M}=1.3 \times 10^{5}, 5 \times 10^{5}$ and $15.5 \times 10^{5}$, the $k_{2} / k_{2}^{0}$ ratios were found to be $1.20,0.79,0.79$ in water and $0.40,0.29,0.18$ in glycol. These results are disturbing on several counts. (a) It is not clear why the rate constant involving the reactive and the shortest catalytic polymer in water solution should actually be higher than for the reaction of their analogues. (b) The decay of $k_{2} / k_{2}^{0}$ with increasing chainlength of the catalytic polymer is much less than predicted by equation 10 . (c) The $k_{2} / k_{2}^{0}$ values are lower in glycol than in water and they decay more rapidly with an increasing chainlength of the catalytic polymer, although the intrinsic viscosities are lower in glycol solution, implying a smaller excluded volume. We are at present unable to account for these discrepancies. We plan to carry out similar studies using polyions as carriers of the reactive and catalytic chain substituents, since the excluded volume can then be varied within wide limits by varying the concentration of simple electrolytes in the systems.

\section{BEHAVIOUR OF CROSSLINKED GELS CARRYING CATALYTIC SUBSTITUENTS}

In recent years, the use of crosslinked polymer gels as chemical reagents has assumed great importance, particularly for the synthesis of polypep- 
tides ${ }^{20,21}$. The work carried out in this area has generally employed commercially available resins as starting materials for the preparation of their reactive derivatives and little attention has been paid to the role which the structure of the crosslinked network may play in optimizing the reactivity of functional groups attached to the polymeric skeleton. The study described below was designed to make a contribution towards the clarification of this problem.

We used as a model reaction the solvolysis of $p$-nitrophenyl-acetate (NPA) and -butyrate (NPB) catalysed by crosslinked acrylamide copolymers containing 10 weight per cent of $N$-acrylyl-4-aminomethylpyridine. The resin was packed into a thermostated column and an aqueous solution of the ester was driven through the resin bed at various speeds. From the dependence of the ester conversion on the contact time a first-order rate constant was calculated and the 'catalytic efficiency' of the resin was defined as the ratio of this rate constant to the rate constant expected in homogeneous solution with the same volume concentration of the catalytic monomer. A preliminary report of the results ohtained has been published ${ }^{22}$. Here I should only like to comment on two features of the data, the dependence of the catalytic efficiency on the degree of crosslinking of the resin, and the topology of the network structure.

Table 1. Dependence of the catalytic efficiency of acrylamide copolymers carrying pendant pyridine residues on the degree of crosslinking (DC). (All polymerizations carried out with a dilution ratio of $1: 1$ of monomer and water).

\begin{tabular}{cccc}
\hline DC, \% & Swelling ratio & NPA & NPB \\
\hline 0.5 & 17.0 & 0.33 & 0.59 \\
1 & 12.4 & 0.74 & 1.5 \\
2 & 7.1 & 1.00 & 4.5 \\
3 & 5.6 & 0.66 & 2.8 \\
4 & 5.0 & 0.21 & 0.91 \\
\hline
\end{tabular}

Table 1 illustrates how the catalytic efficiency of resins prepared by polymerization of the mixed monomers diluted with an equal weight of water depends on the degree of crosslinking. We see that this efficiency passes through a maximum as the degree of crosslinking and the equilibrium swelling of the resin is being varied. The high values of these maxima are particularly remarkable in view of our observation that analogous soluble copolymers have no significant catalytic action on the ester solvolysis. The optimum catalytic efficiency of 4.5 observed in this series with PNB is particularly striking, since it is too large to be accounted for by the concentration of the ester due to partitioning between the aqueous and the resin phase. We believe that the high catalytic efficiency results from a cooperative attack of two pyridine residues on the ester bond. This effect will become less pronounced with increasing swelling of the resin; at very low swelling, on the other hand, the catalytic efficiency decreases due to an unfavourable change in the solvent medium. 
However, it would be a mistake to believe that the swelling ratio of the resin determines uniquely the catalytic efficiency. Table 2 lists results obtained with three resin samples which had swelling ratios in the narrow range of 11.5-13.1, but which differed in the network structure, since the dilution of the monomer during the polymerization was increased at the same time that the crosslink density was raised. There is a pronounced trend towards higher catalytic efficiencies for heavily crosslinked resins prepared at high monomer dilution.

Table 2. Dependence of catalytic efficiency of acrylamide copolymers carrying pendant pyridine residues on the network structure at similar swelling ratios.

\begin{tabular}{ccccc}
\hline DC, $\%$ & $\begin{array}{c}\text { Monomer } \\
\text { dilution ratio }\end{array}$ & $\begin{array}{c}\text { Swelling } \\
\text { ratio }\end{array}$ & \multicolumn{2}{c}{ Catalytic efficiency } \\
NPA & NPB \\
\hline 1 & 1 & 12.4 & 0.74 & 1.5 \\
2 & 2 & 13.1 & 1.8 & 4.3 \\
3 & 3 & 11.5 & 2.6 & 7.2 \\
\hline
\end{tabular}

\section{CONCLUDING REMARKS}

In the preceding discussion I have concentrated on work carried out in our laboratory and I should now like to remark on some related studies carried out elsewhere. Loucheux and Banderet ${ }^{23}$ described an investigation of an intramolecular reaction involving two groups appended, at some distance from each other, to a flexible polymer chain. They treated their data as if the interacting groups could be represented as moving freely within the volume of a sphere hydrodynamically equivalent to the polymer coil. This treatment is clearly faulty, since for a series of polymer chains with a constant density of interacting groups the concentration of these groups within the equivalent sphere would decrease with an increasing polymer chainlength, thus leading to the implausible prediction of a decreasing reaction rate. On the other hand, it is not quite correct to say that the rate of intramolecular interaction of the ends of very long molecular chains cannot be observed. Wang and Davidson ${ }^{24}$ studied the rate of cyclization of a double helical DNA with complementary single strands extending at the two ends of the molecule and they found that their result conformed to equation 2 although this ought to be valid only in the absence of an excluded volume effect. Apparently, these authors were unaware of the complication which an excluded volume (which ought to be substantial for a chain with a high density of ionic charges) might introduce into the theoretical analysis of the intramolecular process. I should also like to refer to the work of Sisido ${ }^{25}$ who has shown that the kinetics of the intramolecular reaction involving reactive and catalytic substituents distributed at random along a chain molecule may be solved analytically, without recourse to computer simulation.

Vollmert and Stutz ${ }^{26}$ studied solutions of two similar copolymers carrying groups which reacted with each other to form a covalent bond. They determined the limiting conversion of the interacting groups and tried to interpret such data in terms of the mutual interpenetration of the molecular coils. This 
treatment cannot be correct, since the formation of a gel structure introduces restraints on the mutual accessibility of the reactive groups which are unrelated to the extent to which the dissolved polymer coils can interpenetrate each other. However, in a study whose results are highly relevant to our investigation, Wetmur and Davidson ${ }^{27}$ measured the kinetics of the renaturation of DNA and found that the rate constant governing the pairing-up of complementary nucleotide residues is subject to a "kinetic excluded volume effect'. This effect was analysed in detail by Wetmur ${ }^{28}$. It would, of course, be expected that such an effect should be particularly large when the two interacting chain molecules are both highly charged.

A cooperative effect of two interacting catalytic groups, which we invoked to explain the high catalytic efficiency of gels carrying nucleophilic substituents, has been demonstrated on a dissolved catalytic polymer by Overberger et al. ${ }^{29}$. A similar mechanism may also have been operative in the study of Kiefer et al. ${ }^{6}$, who reported that imidazole residues appended to polyethylene imine which also carried hydrophobic substituents were $10^{12}$ times more efficient in catalysing the hydrolysis of 4-nitrocatechol sulphate than free imidazole. Even if all the ester molecules are drawn into the polymer domain by hydrophobic interactions, the local concentration of imidazole would not be sufficient to explain this astounding acceleration factor, so that some changes in the reaction mechanism must have been involved.

\section{ACKNOWLEDGEMENT}

I am grateful for financial support of this work by Grant GM-05811 of the National Institutes of Health.

\section{REFERENCES}

1 J. S. Scarpa. D. D. Mueller and 1. M. Klotz, J. Amer. Chem. Soc., 89, 6024 (1967).

2 A. Hvidt and R. Corett, J. Amer. Chem. Soc., 92, 5546 (1970).

${ }^{3}$ H. Ladenheim, E. M. Loebl and H. Morawetz, J. Amer. Chem. Soc, 81, 4860 (1959).

${ }^{4}$ R. L. Letsinger and T. J. Savereide, J. Amer. Chem. Soc., 84, 3127 (1962).

${ }^{5}$ C. G. Overberger, M. Morimoto, I. Cho and J. C. Salamone, J. Amer. Chem. Soc., 93, 3228 (1971).

${ }^{6}$ H. C. Kiefer, N. I. Congdon, I. S. Scarpa and I. M. Klotz, Proc. Natl. Acad. Sci., U.S., 69 , 2155 (1971).

7 E. Gaetjens and H. Morawetz, J. Amer. Chem. Soc, 82, 5328 (1960).

8 T. C. Bruice and U. K. Pandit, J. Amer. Chem. Soc., 82, $5858(1960)$.

9 E. Gaetjens and H. Morawetz, J. Amer, Chem. Soc., 831738 (1961).

${ }_{10}$ W. Kuhn, Kolloid-Z, 68, 2 (1934).

11 P. J. Flory, Statistical Mechanics of Chain Molecules, Interscience Publ., New York, N.Y. (1969).

12 M. E. Fisher, J. Chem. Phys., 45, 1469 (1966).

13 N. Goodman and H. Morawetz, J. Polymer Sci., Pt C, 31, 177 (1970).

14 N. Goodman and H. Morawetz, J. Polymer Sci., Pt A-2,9, 1657 (1971).

15 W. P. Jencks and J. Carriuolo, J. Amer. Chem. Soc., 82, 1778 (1960).

16 H. Morawetz, Macromolecules in Solution, Interscience Publ., New York, N.Y., (1965), p. 433.

17 H. Morawetz and W. R. Song, J. Amer. Chem. Soc., 88, 5714 (1966).

18 H. Morawetz. J.-R. Cho and P. Gans, Macromolecules, 6, 624 (1973).

19 J.-R. Cho and H. Morawetz, Macromolecules, in press.

20 R. B. Merrifield, Advan. Enzymol., 52, 221 (1969). 


\section{REACTIONS OF TWO POLYMER GROUPS}

21 M. Fridkin. A. Patchornik and E. Katchalski, J. Amer. Chem. Soc., 90, 2953 (1968).

22 J. I. Kau and H. Morawetz, Amer. Chem. Soc., Div. Polymer Chem., Polymer Preprints, 13, 819 (1972).

${ }^{23}$ M. H. Loucheux and M. Banderet, J. Polymer Sci, 48, 405 (1960); Bull. Soc. Chim. France, 1964,1220 .

24 J. C. Wang and N. Davidson, J. Mol. Biol., 14, 469 (1960).

25 M. Sisido. Polymer J., 3, 84 (1972).

26 B. Vollmert and H. Stutz. Angew. Makromol. Chem., 3, 182 (1968).

27 J. G. Wetmur and N. Davidson, J. Mol. Biol., 31, 349 (1968).

28 J. G. Wetmur, Biopolymers, 10,601 (1971).

29 C. G. Overberger, T. St. Pierre. N. Vorchheimer, J, Lee and S. Yaroslovsky. J. Amer. Chem. Soc., 87, 296(1965). 